\title{
Optimal diagnostic method using multidetector-row computed tomography for predicting lymph node metastasis in colorectal cancer
}

Tsutomu Kumamoto ${ }^{1,4^{*}}$ D, Junichi Shindoh ${ }^{2}$, Hideaki Mita ${ }^{1}$, Yuriko Fujiii ${ }^{3}$ Yuichiro Mihara ${ }^{1}$, Michiro Takahashi ${ }^{1}$, Nobuyuki Takemura', Takako Shirakawa ${ }^{3}$, Hisashi Shinohara ${ }^{4}$ and Hiroya Kuroyanagi ${ }^{2}$

\begin{abstract}
Background: Prediction of nodal involvement in colorectal cancer is an important aspect of preoperative workup to determine the necessity of preoperative treatment and the adequate extent of lymphadenectomy during surgery. This study aimed to investigate newer multidetector-row computed tomography (MDCT) findings for better predicting lymph node $(\mathrm{LN})$ metastasis in colorectal cancer.

Methods: Seventy patients were enrolled in this study; all underwent MDCT prior to surgery and upfront curative resection for colorectal cancer. LNs with a short-axis diameter (SAD) $\geq 4 \mathrm{~mm}$ were identified on MDCT images, and the following measures were recorded by two radiologists independently: two-dimensional (2D) SAD, 2D long-axis diameter ( $L A D), 2 D$ ratio of $S A D$ to $L A D, 2 D C T$ attenuation value, three-dimensional (3D) SAD, 3D LAD, 3D SAD to LAD ratio, 3D CT attenuation value, LN volume, and presence of extranodal neoplastic spread (ENS), as defined by indistinct nodal margin, irregular capsular enhancement, or infiltration into adjacent structures.
\end{abstract}

Results: Forty-six patients presented $173 \mathrm{LNs}$ with a SAD $\geq 4 \mathrm{~mm}$, while 24 patients exhibited pathologically confirmed LN metastases. Receiver operating characteristic analysis revealed that 2D LAD was the most sensitive measure for LN metastases with an area under the curve of 0.752 (cut-off value, $7.05 \mathrm{~mm}$ ). When combined with CT findings indicating ENS, 2D LAD (> or $\leq 7 \mathrm{~mm}$ ) showed enhanced predictive power for LN metastases (area under the curve, $0.846 ; p<0.001$ ).

Conclusions: $L A D$ in axial MDCT imaging is the most sensitive measure for predicting colorectal LN metastases, especially when MDCT findings of ENS are observed.

Keywords: Colorectal cancer, Lymph node metastasis, Multidetector-row computed tomography, Preoperative diagnosis

\section{Background}

Colorectal cancer is the third leading cause of cancer-related death worldwide. The presence of lymph node (LN) metastasis is one of the most important factors associated with poor prognosis $[1,2]$, and preoperative treatment is considered in selected patients who are

\footnotetext{
* Correspondence: k-s-tomu@amber.plala.or.jp

'Department of Gastrointestinal Surgery, JR Tokyo General Hospital, Yoyogi

2-1-3, Shibuya-ku, Tokyo, Japan

${ }^{4}$ Present Address: Department of Surgery, Hyogo College of Medicine,

Mukogawa-cho 1-1, Nishinomiya, Hyogo 663-8501, Japan

Full list of author information is available at the end of the article
}

expected to exhibit prolonged survival $[3,4]$. Since preoperative staging is critical in determining treatment sequence and surgical indication, preoperative prediction of nodal involvement is an important aspect of colorectal cancer management.

The LNs measuring $>10 \mathrm{~mm}$ in computed tomography $(\mathrm{CT})$ images have conventionally been regarded as a sign of nodal involvement of cancer [5, 6]. Previous studies have reported the diagnostic accuracy of such CT measures, using axial images [5-9]. However, these reports were based on data collected prior to the

(c) The Author(s). 2019 Open Access This article is distributed under the terms of the Creative Commons Attribution 4.0 International License (http://creativecommons.org/licenses/by/4.0/), which permits unrestricted use, distribution, and 
availability of current high-resolution CT imaging equipment; therefore, the predictive value of CT findings for LN metastasis has not yet been fully validated.

Recently, use of multidetector-row computed tomography (MDCT) has become a routine practice for preoperative diagnosis, as its thinner slice data enable various additional analyses, including three-dimensional (3D) reconstruction. However, it remains unclear how much this novel imaging modality has changed the accuracy of cancer diagnosis.

In this study, we evaluated the diagnostic accuracy of various radiologic measures obtained by newer MDCT data and sought to re-evaluate a sensitive approach to predict nodal involvement in colorectal cancer.

\section{Methods \\ Patients}

This study was approved by the Ethics Committee of our hospital. Our prospectively collected colorectal cancer database was analyzed retrospectively. The initial population considered for this study included 95 consecutive patients who underwent preoperative MDCT and resection of colorectal cancer at our institution between April 2016 and February 2018. Of these, the following patients were excluded from the current analysis in order to analyze the pure correlation between CT images and pathologic findings: patients with non-curative resection $(n=11)$, patients who underwent preoperative chemotherapy and/or radiation therapy $(n=6)$, patients for whom the current CT protocol was not followed ( $n$ $=6$ ), and patients who did not exhibit a one-to-one correlation between detected LNs and dissected LNs $(n=2)$. The remaining 70 patients were studied in detail; all remaining patients had been diagnosed with adenocarcinoma of colorectal origin and had undergone complete mesocolic excision or total mesorectal excision with central vascular ligation $[10,11]$.

\section{CT protocol}

A 64-detector row CT scanner (OPTIMA CT660PRO; GE Healthcare, Chicago, IL, USA) was used for CT analysis. Protocol CT parameters were as follows: section thickness, $0.625 \mathrm{~mm}$; pitch, 0.984; construction interval, $0.625 \mathrm{~mm}$; noise index, 9.8 (at 5-mm thickness); $120 \mathrm{kV}$; and tube rotation time, $0.7 \mathrm{~s}$. A total of $60 \mathrm{mg}$ I per $\mathrm{kg}$ of non-ionic contrast material was administered intravenously over a period of $30 \mathrm{~s}$, using a power injector (Stellant CT Injection System Stellant D Dural Flow; Bayer Medical Care, Pittsburgh, PA, USA). Imaging was performed at 25 and $85 \mathrm{~s}$ after the initiation of contrast material injection, corresponding to the arterial and late phases, respectively.

\section{Evaluation}

The LNs were screened on axial MDCT images (0.625-mm-thick slices); the mediastinal window settings consisted of a window level of 55 and a window width of 300 , in the late phase. Two experienced radiologists identified enlarged LNs measuring $\geq 4 \mathrm{~mm}$ in short-axis diameter (SAD), preoperatively. The following CT measures were recorded in every patient: long-axis diameter (LAD), the ratio of SAD to $\mathrm{LAD}$, and maximum and average CT attenuation values. The presence of indistinct nodal margins, irregular nodal capsular enhancement, or infiltration into the adjacent structure (Fig. 1) were also recorded as signs of "extranodal neoplastic spread" (ENS) [12]. In addition, all LNs were reconstructed in 3D using a workstation (Advantage Workstation Volume Share 4, version 4.5, GE Healthcare, Milwaukee, WI, USA); 3D SAD, 3D LAD, maximum and average $\mathrm{CT}$ attenuation values in $3 \mathrm{D}$ images, and $\mathrm{LN}$ volume were also measured (Fig. 2). For each LN, a one-to-one correlation between the detected and dissected LN was confirmed; the LN was then assessed pathologically.

\section{Statistical analysis}

All statistical analyses were performed using SPSS version 24. (IBM Corp., Armonk, NY, USA). Factors associated with LN enlargement on MDCT were investigated using multivariate logistic regression with backward elimination. The predictive value of $\mathrm{LN}$ metastases was measured via area under the curve (AUC) in a receiver operating characteristic curve analysis. The optimal cut-off point in the receiver operating characteristic

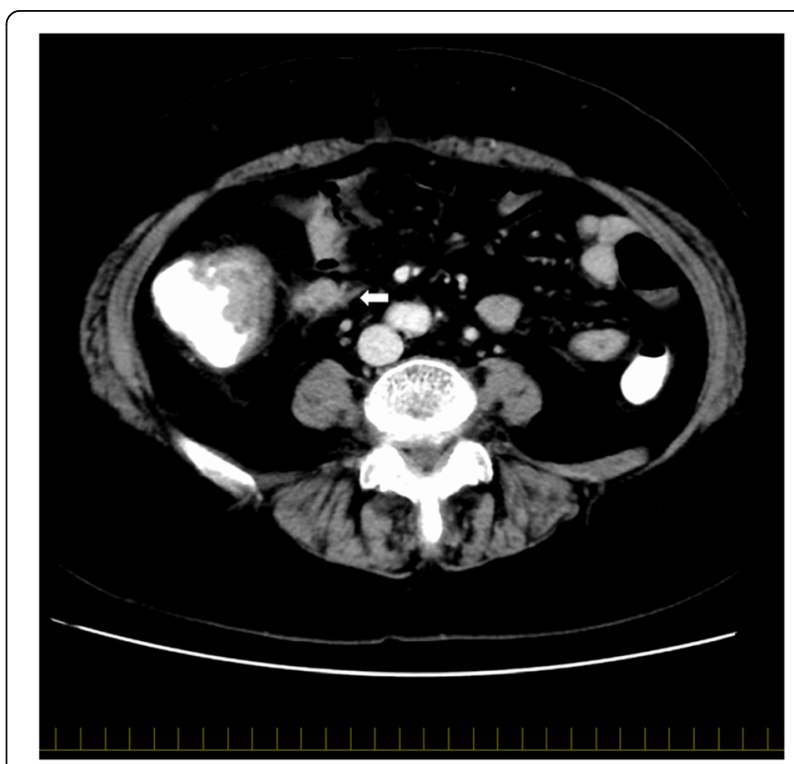

Fig. 1 Extranodal neoplastic spread (ENS): the white arrow indicates a lymph node with ENS 


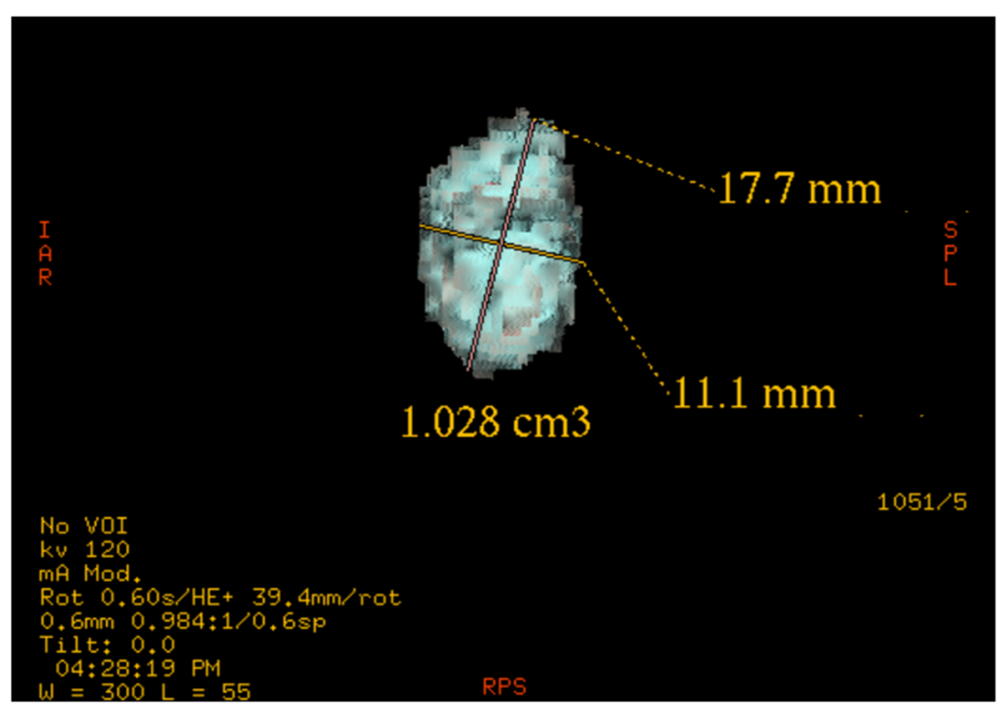

Fig. 2 Three-dimensional reconstruction: the size and volume of the lymph node were measured

analysis was determined by Youden's index (J), calculated using the equation $J=$ sensitivity $+(1-$ specificity $)$.

\section{Results}

\section{Patients and LN status}

The baseline characteristics of the patient population are summarized in Table 1. In total, 1644 LNs were dissected from 70 patients (median, $21.5 \mathrm{LNs}$; range, 4-50 LNs per patient). A total of 173 enlarged LNs were detected in 46 patients. Of these, 56 LNs (32.4\%) from 24 (52.2\%) patients were histopathologically positive for LN metastasis. In another four patients who did not show LN enlargement on preoperative MDCT, LN metastasis was confirmed pathologically.

\section{Factors influencing LN enlargement on MDCT}

Table 2 shows the results of univariate analysis for factors predicting LN enlargement. Bowel obstruction, increased white blood cell count, and pathologically confirmed metastasis correlated with LN enlargement, as detected on MDCT. Multivariate analysis revealed that pathologically confirmed metastasis was the only predictive factor for LN enlargement (odds ratio, 6.00; 95\% confidence interval, 2.08-17.3; $p=0.006$ ).

\section{Evaluation of LN enlargement on MDCT}

The comparison of the predictive power of various CT measures for histopathologic nodal involvement of cancer is shown in Fig. 3. The 2D LAD on the axial image showed the highest AUC (0.752), followed by 2D SAD (AUC, 0.742), nodal volume (AUC, 0.717), 3D SAD (AUC, 0.705), 3D LAD (AUC, 0.698), 3D average CT attenuation value (AUC, 0.659), 3D maximum $C T$ value (AUC, 0.654), 2D maximum CT attenuation value (AUC,
0.599), 3D size ratio (AUC, 0.511), 2D size ratio (AUC, 0.503 ), and $2 \mathrm{D}$ average $\mathrm{CT}$ attenuation value (AUC, 0.438). The optimal cut-off value of 2D LAD for predicting LN metastases was $7.05 \mathrm{~mm}$, and the sensitivity, 0.821 ; specificity, 0.624 ; accuracy, 0.688 ; positive predictive value (PPV), 0.511; and negative predictive value (NPV), 0.880 were estimated.

Among the 173 enlarged LNs, ENS was observed in 41 (23.7\%). The predictive power for LN metastases, focusing on these LNs presenting ENS, was superior to that for the simple morphological enlargement of LNs (sensitivity, 0.643; specificity, 0.957; accuracy, 0.855; PPV, 0.878; and NPV, 0.848).

Based on these results, a new scoring system was developed: 2D LAD $(>7 \mathrm{~mm}[1$ point $]$ or $\leq 7 \mathrm{~mm}[0$ point $]$ ) + ENS (positive [ 1 point] or negative [ 0 point $]$ ). The probability of histopathologic metastases was $91.7 \%$ when both criteria were met ( 2 points), $27.1 \%$ when one of these criteria was not met (1 point), and $9.0 \%$ when neither criterion was met (0 points) (Table 3$)$. The AUC of this new scoring system was 0.846 .

\section{Potential influence of sidedness of colorectal cancer}

When the population was subclassified into left and right, in accordance with the location of the primary tumor, the size of 2D LAD for right-sided primary tumors was larger than that for left-sided primary tumors $(p<0.005)$ (median, $8.25 \mathrm{~mm}$; range, $4.55-21.10$ $\mathrm{mm}$ vs. median, $6.68 \mathrm{~mm}$; range, $4.70-22.05 \mathrm{~mm}$, respectively), while the incidence of histopathologic metastases was similar between right-sided and left-sided primary tumors $(37.9 \%$ vs. $25.6 \%, p=0.103)$. The AUCs for 2D LAD and the scoring system were 0.691 (cut-off value, $7.15 \mathrm{~mm}$ ) and 0.842 , respectively, for 
Table 1 Baseline patient characteristics

\begin{tabular}{|c|c|}
\hline Characteristic & Value $(n=70)$ \\
\hline Age (years) & 70 (39-95) \\
\hline Male/female ratio & $41 / 29$ \\
\hline Body mass index $\left(\mathrm{kg} / \mathrm{m}^{2}\right)$ (range) & $22.6(14.1-30.5 \%)$ \\
\hline \multicolumn{2}{|l|}{ Tumor location } \\
\hline Appendix vermiformis/cecum & $15(21.4 \%)$ \\
\hline Ascending colon & $9(12.9 \%)$ \\
\hline Transverse colon & $8(11.4 \%)$ \\
\hline Descending colon & $3(4.3 \%)$ \\
\hline Sigmoid colon/rectosigmoid colon & $25(35.7 \%)$ \\
\hline Rectum & $10(14.3 \%)$ \\
\hline \multicolumn{2}{|l|}{ Depth of tumor } \\
\hline Mucosa & $4(5.7 \%)$ \\
\hline Submucosa & $14(20.0 \%)$ \\
\hline Muscularis propria & $9(12.9 \%)$ \\
\hline Subserosa & $28(40.0 \%)$ \\
\hline $\begin{array}{l}\text { Exposure on submucosa or invade } \\
\text { other organs or structures }\end{array}$ & $15(21.4 \%)$ \\
\hline \multicolumn{2}{|l|}{ Differentiation $^{\mathrm{a}}$} \\
\hline Well differentiated & $32(45.7 \%)$ \\
\hline Moderately differentiated & $35(50.0 \%)$ \\
\hline Poorly differentiated & $3(4.3 \%)$ \\
\hline Obstruction $^{\mathrm{b}}$ & $17(24.3 \%)$ \\
\hline Preoperative intervention & $7(10.0 \%)$ \\
\hline Endoscopic resection & $4(5.7 \%)$ \\
\hline lleus tube & $3(4.3 \%)$ \\
\hline $\mathrm{WBC}\left(/ \mathrm{mm}^{3}\right)$ & $5700(2600-14,700)$ \\
\hline NLR & $2.40(0.01-9.65)$ \\
\hline $\mathrm{CRP}(\mathrm{mg} / \mathrm{mL})$ & $0.16(0.01-6.20)$ \\
\hline CEA (ng/mL) & $3.6(0.7-98)$ \\
\hline CA19-9 (U/L) & $6.0(0.07-4412)$ \\
\hline Days from $\mathrm{CT}$ to surgery & $20(2-71)$ \\
\hline Lymph node enlargement on CT & $46(65.7 \%)$ \\
\hline Pathologically confirmed metastasis & $28(40.0 \%)$ \\
\hline
\end{tabular}

Data are presented as median (range) or number (\%) unless otherwise indicated

WBC white blood cell, NLR neutrophil to lymphocyte ratio, CRP c-reactive protein, CEA carcinoembryonic antigen, CA19-9 carbohydrate antigen 19-9, CT computed tomography

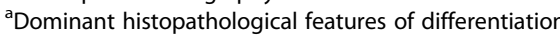

${ }^{b}$ Obstruction, which was not passed through with an endoscope

right-sided primary tumors, while the AUCs were 0.805 (cut-off value, $7.05 \mathrm{~mm}$ ) and 0.844 , respectively, for left-sided primary tumors.

\section{Discussion}

The current study sought the best CT measure for predicting LN metastases from colorectal cancer. Among the various measures available on MDCT, 2D LAD was
Table 2 Univariate analyses of clinical factors predicting lymph node enlargement on multidetector computed tomography

\begin{tabular}{ll}
\hline Clinical factor & $p$ value \\
\hline Age & 0.556 \\
Male/female ratio & 0.630 \\
Body mass index & 0.586 \\
Tumor location (right side vs left side) & 0.623 \\
Differentiation & 0.323 \\
Obstruction & 0.025 \\
lleus tube & 0.201 \\
Endoscopic resection & 0.077 \\
WBC & 0.004 \\
NLR & 0.138 \\
CRP & 0.204 \\
Pathologically confirmed metastasis & 0.004 \\
\hline
\end{tabular}

WBC white blood cell, NLR neutrophil to lymphocyte ratio, CRP c-reactive protein

the most sensitive measure; moreover, the presence of ENS was associated with LN metastases. Although the baseline sizes of LNs seemed to be larger in right-sided primary tumors, the scoring system using 2D LAD and ENS showed good performance for predicting LN metastases from primary tumors on either side.

Conventionally, a LAD of $>10 \mathrm{~mm}$ has been accepted as a potential sign of LN metastases [5, 6]. Reported sensitivities and specificities of LN size and/or clusters of $\geq 3 \mathrm{LNs}$ were $56-84.3 \%$ and 58-95\%, respectively [5-9]. Dighe et al. demonstrated that studies using $\leq 5$-mm-thick CT sections show significantly better results than those using > 5-mm-thick CT sections [13]; moreover, a recent retrospective study reviewed the accuracy of conventional LN size criteria, using recent $\mathrm{CT}$ techniques, and reported that the ability of preoperative CT to assess LNs remained unsatisfactory [14].

In the current study, various radiologic measures available in MDCT were compared to establish a valid method for the prediction of LN metastases. Among the tested CT measures, 2D LAD in axial images showed the best performance for the prediction of LN metastases; notably, 3D reconstruction did not show diagnostic superiority to the $2 \mathrm{D}$ measures. Interestingly, although the baseline size of LNs seemed to be larger in right-sided primary tumors, the cut-off values for $2 \mathrm{D}$ LAD were similar between the right-sided and left-sided primary tumors $(7.15 \mathrm{~mm}$ vs. $7.05 \mathrm{~mm})$.

The current study also tested the diagnostic accuracy of $\mathrm{CT}$ attenuation and size ratio (SAD to LAD). A previous study reported that the cut-off value of 0.8 in size ratio was associated with an increased incidence of LN metastases [15], while another study reported that a measurement of $\geq 100$ Hounsfield units was correlated with LN metastases [9]. However, these measures did 


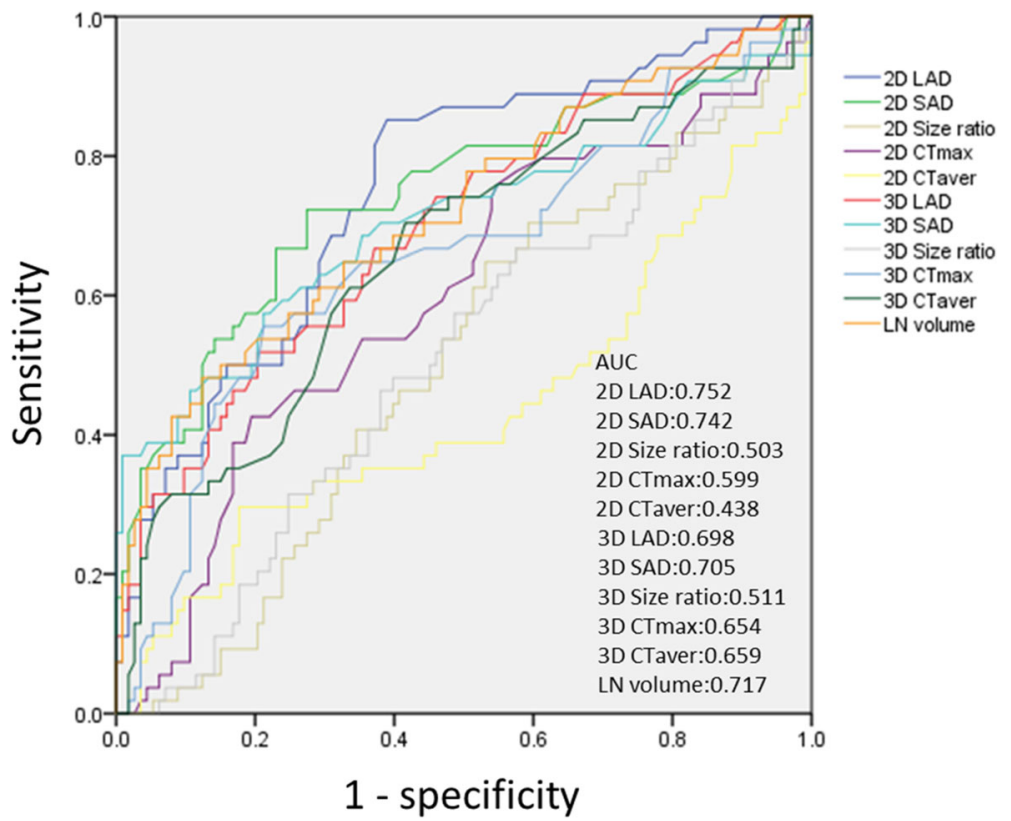

Fig. 3 Receiver operating characteristic curves show the ability of each multidetector computed tomography measurement to predict lymph node metastasis. AUC, area under the curve; 2D, two-dimensional; 3D, three-dimensional; SAD, short-axis diameter; LAD, long-axis diameter; CTmax, maximum computed tomography attenuation value; CTaver, average computed tomography attenuation value; LN, lymph node

not show good performance in the present study. However, a new measure, ENS, showed good correlation with LN metastases in this study. ENS indicates a certain type of morphology and is one of the criteria for diagnosing LN metastasis in cervical nodes from head and neck squamous cell carcinoma [12]. A recent retrospective study also reported that the presence of at least one LN with internal heterogeneity and/or irregular outer border is an important predictive factor in colon cancer [16]. Given that 2D LAD and ENS showed good correlation with the incidence of LN metastases, a scoring system was established in this study. This simple score, using a 7-mm cut-off value for 2D LAD, along with the presence or absence of ENS, showed good predictive performance for LN metastases, even in the subgroup analysis stratified by the sidedness of the primary tumor.

The limitations of this study include its retrospective nature and the relatively small patient sample size. However, the current analysis used prospectively corrected $\mathrm{CT}$ findings measured by two independent radiologists;

Table 3 Predictive probability for histopathologic metastases in the scoring system generated in this study

\begin{tabular}{lll}
\hline Score & $\begin{array}{l}\text { Number of LN } \\
\text { metastases (\%) }\end{array}$ & $\begin{array}{l}\text { Number of non-LN } \\
\text { metastases (\%) }\end{array}$ \\
\hline Score 0 & $7(9.0 \%)$ & $71(91.0 \%)$ \\
Score 1 & $16(27.1 \%)$ & $43(72.9 \%)$ \\
Score 2 & $33(91.7 \%)$ & $3(8.3 \%)$ \\
\hline
\end{tabular}

moreover, a sufficient number of LNs were studied, with a one-to-one correlation of the LNs detected on CT to those confirmed pathologically. Although validation studies using a larger population are needed, the current results may offer a simple and potentially reliable method to predict LN metastases in colorectal cancer.

\section{Conclusions}

In conclusion, 2D LAD shows the best performance in predicting LN metastases; evaluation for the presence of ENS further improves the diagnostic accuracy for predicting LN metastases prior to surgery. A prospective study to validate the current simple scoring system, using 2D LAD and ENS, is therefore warranted.

\section{Abbreviations \\ AUC: Area under the curve; CT: Computed tomography; ENS: Extranodal neoplastic spread; LAD: Long-axis diameter; LN: Lymph node; \\ MDCT: Multidetector-row computed tomography; ROC: Receiver operating characteristic; SAD: Short-axis diameter}

\section{Acknowledgements}

We thank Hitoshi Nagano and Takuya Kawasaki for their advice regarding the $C T$ protocol and settings.

\section{Funding}

No funding was received for this research and/or publication.

\section{Availability of data and materials}

All data generated or analyzed during this study are included in this published article. 


\section{Authors' contributions}

TK was the guarantor of integrity of the entire study; the study concept and design were developed by TK, JS, and TS; the data collection and analysis were performed by TK, HM, YF, YM, MT, NT, and TS; the statistical analysis was performed by TK and JS; the manuscript was prepared by TK and JS; and manuscript editing was performed by HS and HK. All authors read and approved the final manuscript.

\section{Ethics approval and consent to participate}

This study was approved by the Ethics Committee of JR Tokyo General Hospital.

\section{Consent for publication}

Not applicable.

\section{Competing interests}

The authors declare that they have no competing interests.

\section{Publisher's Note}

Springer Nature remains neutral with regard to jurisdictional claims in published maps and institutional affiliations.

\section{Author details}

'Department of Gastrointestinal Surgery, JR Tokyo General Hospital, Yoyogi 2-1-3, Shibuya-ku, Tokyo, Japan. '2Department of Gastroenterological Surgery, Toranomon Hospital, Tranomon 2-2-2, Minato-ku, Tokyo, Japan. ${ }^{3}$ Department of Radiology, JR Tokyo General Hospital, Yoyogi 2-1-3, Shibuya-ku, Tokyo, Japan. ${ }^{4}$ Present Address: Department of Surgery, Hyogo College of Medicine, Mukogawa-cho 1-1, Nishinomiya, Hyogo 663-8501, Japan.

Received: 14 December 2018 Accepted: 15 February 2019

Published online: 22 February 2019

\section{References}

1. Watanabe T, Itabashi M, Shimada Y, Tanaka S, Ito Y, Ajiokaet Y, et al. Japanese Society for Cancer of the Colon and Rectum (JSCCR) Guidelines 2014 for treatment of colorectal cancer. Int J Clin Oncol. 2015;20:207-39.

2. MERCURY Study Group, Shihab OC, Taylor F, Bees N, Blake H, Jeyadevan N, et al. Relevance of magnetic resonance imaging-detected pelvic sidewall lymph node involvement in rectal cancer. Br J Surg. 2011;98:1798-104.

3. Chau I, Chan S, Cunningham D. Overview of perioperative and postoperative therapy for colorectal cancer: the European and United States perspectives. Clin Colorectal Cancer. 2003;3:19-33.

4. Roh MS, Colangelo LH, O'Connell MJ, Yothers G, Deutsch M, Allegra CJ, et al. Preoperative multimodality therapy improves disease-free survival in patients with carcinoma of rectum: NSABP R-03. J Clin Oncol. 2009; 27:5124-30

5. Harvey CJ, Amin Z, Hare CM, Gillams AR, Novelli MR, Boulos PB, et al. Helical $C T$ pneumocolon to assess colonic tumors: radiologic-pathologic correlation. AJR Am J Roentgenol. 1998;170:1439-43.

6. Gomille T, Aleksic M, Ulrich B, Christ F. Significance of $C T$ in the detection of regional lymph node metastases in colorectal carcinoma. Radiologe. 1998; 38:1077-82.

7. Acunaş B, Rozanes I, Acunas G, Celik L, Sayi I, Gökmen E. Preoperative CT staging of colon carcinoma (excluding the recto-sigmoid region). Eur J Radiol. 1990:11:150-3.

8. Balthazar EJ, Megibow AJ, Hulnick D, Naidich DP. Carcinoma of the colon: detection and preoperative staging by CT. AJR Am J Roentgenol. 1988;150: $301-6$.

9. Hundt W, Braunschwieg R, Reiser M. Evaluation of spiral CT in staging of colon and rectum carcinoma. Eur Radiol. 1999;9:78-84.

10. Hohenberger W, Weber K, Matzel K, Papadopoulos T, Merkel S. Standardized surgery for colonic cancer: complete mesocolic excision and central ligation-technical notes and outcome. Color Dis. 2009;11:354-64.

11. Heald RJ, Ryall RD. Recurrence and survival after total mesorectal excision for rectal cancer. Lancet. 1986;1:1479-82.

12. King AD, Tse GM, Yuen EH, To EW, Vlantis AC, Zee B, et al. Comparison of $C T$ and MR imaging for the detection of extra nodal spread in metastatic neck nodes. Eur J Radiol. 2004;52:264-70.

13. Dighe S, Purkayastha S, Swift I, Tekkis PP, Darzi A, A'Hern R, et al. Diagnostic precision of $\mathrm{CT}$ in local staging colon cancers: a meta-analysis. Clin Radiol. 2010;65:708-19.
14. de Vries FE, da Costa DW, van der Mooren K, van Dorp TA, Vrouenraets BC. The value of pre-operative computed tomography scanning for the assessment of lymph node status in patients with colon cancer. Eur J Surg Oncol. 2014:40:1777-81.

15. Kanamoto T, Matsuki M, Okuda J, Inada Y, Tatsugami F, Tanikake M, et al. Preoperative evaluation of local invasion and metastatic lymph nodes of colorectal cancer and mesenteric vascular variations using multidetectorrow computed tomography before laparoscopic surgery. J Comput Assist Tomogr. 2007;31:831-9.

16. Rollvén E, Abraham-Nordling M, Holm T, Blomqvist L. Assessment and diagnostic accuracy of lymph node status to predict stage III colon cancer using computed tomography. Cancer Imaging. 2017;17:3.

\section{Ready to submit your research? Choose BMC and benefit from:}

- fast, convenient online submission

- thorough peer review by experienced researchers in your field

- rapid publication on acceptance

- support for research data, including large and complex data types

- gold Open Access which fosters wider collaboration and increased citations

- maximum visibility for your research: over $100 \mathrm{M}$ website views per year

At $\mathrm{BMC}$, research is always in progress.

Learn more biomedcentral.com/submissions 